6-1-2004

\title{
Comparison of transcranial electric motor and somatosensory evoked potential monitoring during cervical spine surgery.
}

\author{
Alan S Hilibrand \\ Rothman Institute, Thomas Jefferson University \\ Daniel M Schwartz \\ Surgical Monitoring Associates \\ Venkat Sethuraman \\ Rothman Institute, Thomas Jefferson University \\ Alexander R Vaccaro \\ Rothman Institute, Thomas Jefferson University \\ Todd J Albert \\ Rothman Institute Thomas Jefferson University \\ Follow this and additional works at: https://jdc.jefferson.edu/rothman_institute \\ Part of the Orthopedics Commons \\ Let us know how access to this document benefits you
}

\section{Recommended Citation}

Hilibrand, Alan S; Schwartz, Daniel M; Sethuraman, Venkat; Vaccaro, Alexander R; and Albert, Todd J, "Comparison of transcranial electric motor and somatosensory evoked potential monitoring during cervical spine surgery." (2004). Rothman Institute Faculty Papers. Paper 10. https://jdc.jefferson.edu/rothman_institute/10

This Article is brought to you for free and open access by the Jefferson Digital Commons. The Jefferson Digital Commons is a service of Thomas Jefferson University's Center for Teaching and Learning (CTL). The Commons is a showcase for Jefferson books and journals, peer-reviewed scholarly publications, unique historical collections from the University archives, and teaching tools. The Jefferson Digital Commons allows researchers and interested readers anywhere in the world to learn about and keep up to date with Jefferson scholarship. This article has been accepted for inclusion in Rothman Institute Faculty Papers by an authorized administrator of the Jefferson Digital Commons. For more information, please contact: JeffersonDigitalCommons@jefferson.edu. 


\title{
COMPARISON OF TRANSCRANIAL Electric Motor and SOMATOSEnSORY Evoked Potential Monitoring During Cervical Spine Surgery
}

\author{
By Alan S. Hilibrand, MD, DANiEl M. SCHWARTZ, PHD, DABNM, \\ Venkat SETHuraman, MD, Alexander R. VACCARO, MD, AND TODD J. Albert, MD
}

Investigation performed at Surgical Monitoring Associates, Bala Cynwyd, and The Rothman Institute, Philadelphia, Pennsylvania

\begin{abstract}
Background: There has been little enthusiasm for somatosensory evoked potential monitoring in cervical spine surgery as a result, in part, of the increased risk of motor tract injury at this level, to which somatosensory monitoring may be insensitive. Transcranial electric motor evoked potential monitoring allows assessment of the motor tracts; therefore, we compared transcranial electric motor evoked potential and somatosensory evoked potential monitoring during cervical spine surgery to determine the temporal relationship between the changes in the potentials demonstrated by each type of monitoring and neurological sequelae and to identify patient-related and surgical factors associated with intraoperative neurophysiological changes.
\end{abstract}

Methods: Somatosensory evoked potential and transcranial electric motor evoked potential data recorded for 427 patients undergoing anterior or posterior cervical spine surgery between January 1999 and March 2001 were analyzed. All patients who showed substantial (at least 60\%) or complete unilateral or bilateral amplitude loss, for at least ten minutes, during the transcranial electric motor evoked potential and/or somatosensory evoked potential monitoring were identified.

Results: Twelve of the 427 patients demonstrated substantial or complete loss of amplitude of the transcranial electric motor evoked potentials. Ten of those patients had complete reversal of the loss following prompt intraoperative intervention, whereas two awoke with a new motor deficit. Somatosensory evoked potential monitoring failed to identify any change in one of the two patients, and the change in the somatosensory evoked potentials lagged behind the change in the transcranial electric motor evoked potentials by thirty-three minutes in the other. No patient showed loss of amplitude of the somatosensory evoked potentials in the absence of changes in the transcranial electric motor evoked potentials. Transcranial electric motor evoked potential monitoring was 100\% sensitive and $100 \%$ specific, whereas somatosensory evoked potential monitoring was only $25 \%$ sensitive; it was, however, $100 \%$ specific.

Conclusions: Transcranial electric motor evoked potential monitoring appears to be superior to conventional somatosensory evoked potential monitoring for identifying evolving motor tract injury during cervical spine surgery. Surgeons should strongly consider using this modality when operating on patients with cervical spondylotic myelopathy in general and on those with ossification of the posterior longitudinal ligament in particular.

Level of Evidence: Diagnostic study, Level I-1 (testing of previously developed diagnostic criteria in series of consecutive patients [with universally applied reference "gold" standard]). See Instructions to Authors for a complete description of levels of evidence.

$\mathrm{N}$ europhysiological monitoring is used during spine surgery to assess the function of the spinal cord and to identify any evolving iatrogenic spinal cord injury. Studies of patients undergoing surgery for scoliosis and thoracolumbar injuries have demonstrated that continuous somatosensory evoked potential (SSEP) monitoring can reduce the risk of iatrogenic paraplegia ${ }^{1-7}$. Despite the success of identifi- cation of iatrogenic injury with SSEP monitoring during operations for the correction of scoliosis and other corrective operations on the thoracic spine, SSEPs are mediated primarily by the dorsal sensory spinal cord tracts. As such, the diagnosis of an impending motor tract injury based on changes in SSEP amplitude and/or latency is presumptive at best. Hence, there is the risk of a false-negative result ${ }^{8-13}$. 
The Journal of Bone \& JOINT Surgery • JBIS. ORG VOLUME 86-A · NUMBER 6 - JUNE 2004
Transcranial EleCtRIC MOTOR AND SOMATOSENSORY Evoked Potentials in Cervical Spine Surgery
Although transcranial electric motor evoked potential (tceMEP) monitoring has shown great promise with regard to more rapid identification of corticospinal tract injury during excision of spinal cord tumors or correction of scoliosis ${ }^{14-20}$, there is a dearth of information related to its use to monitor motor tract function during cervical spine surgery ${ }^{21,22}$. Hence, this study was designed to determine and compare the sensitivity and specificity of tceMEP and conventional SSEP monitoring for detection of impending spinal cord injury during cervical spine surgery; to determine the temporal relationship between the changes demonstrated by these two monitoring modalities and subsequent, clinically important neurological sequelae, and to identify patient-related and surgical risk factors associated with intraoperative changes in these evoked potentials.

\section{Materials and Methods}

The protocol for this study was reviewed by the institu1 tional review board of Thomas Jefferson University Hospital and was granted an exempt status because it was an anonymous retrospective chart review. Outcomes data for all cervical spine surgical procedures performed with multimodality spinal cord monitoring between January 1, 1999, and March 31, 2001, at a single institution were reviewed. A total of 427 procedures were performed in 242 male patients (57\%) and 185 female patients (43\%) ranging in age from fifteen to ninety-five years, with an average age of fifty years, at the time of the index procedure. There were 324 anterior, eighty-three posterior, and twenty combined anterior and posterior procedures. Of the 427 patients, 216 (51\%) presented with a preoperative diagnosis of cervical spondylotic myelopathy, and twenty-two (10\%) of the 216 had ossification of the posterior longitudinal ligament. Intraoperative records were examined in an attempt to identify the operative event that correlated with the neurophysiologic change as well as the effect of surgical and/or anesthesia-related intervention on the changes demonstrated by monitoring. Hospital and office charts were also reviewed to determine the preoperative diagnoses as well as the preoperative, immediate postoperative, and most recent neurological data. The anesthesia protocol used during the study period is described in detail in the Appendix.

\section{Spinal Cord Monitoring}

Spinal cord monitoring was performed continuously, from the time of induction of anesthesia until the patient emerged from the anesthesia, by recording both upper and lower-extremity efferent transcranial electric motor (tceMEPs) and afferent somatosensory evoked potentials (SSEPs). Cortical and subcortical SSEPs were elicited to a $300-\mu S$ square-wave electrical pulse presented sequentially to the posterior tibial and ulnar nerves at a rate of $4.7 / \mathrm{sec}$. Stimulation intensity levels ranged between 35 and $50 \mathrm{~mA}$. These levels were selected as being well within the asymptotic portion of the SSEP intensity versus amplitude plot for each patient. Cortical potentials were recorded from gold-plated cup electrodes (Grass Instruments, Quincy, Massachusetts) affixed to Cpz, Cp3, and Cp4 and ref- erenced to Fpz (international 10-20 system). Subcortical cervical/brainstem responses were recorded over the surface of the C2 or C3 vertebra and also referenced to Fpz. Commercially available neurophysiology instrumentation (Nicolet Endeavor; Nicolet Biomedical, Madison, Wisconsin, or Cadwell Sierra; Cadwell Laboratories, Kennewick, Washington) was used for all SSEP stimulation and recording.

Transcranial electric motor evoked potentials were recorded over the first dorsal interosseous muscle in the upper extremities and both tibialis anterior and abductor hallucis muscles in the lower extremities following a brief-duration, high-voltage (400 to $1000-\mathrm{V}$ ) anodal electrical stimulus train (pulse width $=50 \mu \mathrm{S}, \mathrm{N}=3$ to 7 , interpulse interval $=1$ to 5 $\mathrm{msec})$. The multipulse stimulus was delivered between two corkscrew-type electrodes (A-Gram, Glenn Rock, New Jersey) inserted over motor cortex regions at C1 and C2 (international 10-20 system). Stimuli were delivered through a commercially available transcortical stimulator (D185; Digitimer, Welwyn Garden City, United Kingdom) with responses recorded on the same system used for monitoring SSEPs.

\section{Response Interpretation}

A clinically relevant neurophysiological change was defined as an intraoperative unilateral or bilateral amplitude loss of at least $60 \%$ with persistence over at least a ten-minute duration. This cutoff value, equating to a greater than 2.0 standard deviation criterion for a major change, was selected to reduce the possibility of false-positive interpretation due to response variability, as reported by York et al. ${ }^{23}$. If such a change in evoked potentials was detected, anesthesia personnel were directed to increase the patient's mean arterial pressure to at least $90 \mathrm{~mm} \mathrm{Hg}$. A failure of the response amplitude to improve over the course of five to ten minutes led to the administration of so-called spinal-cord-injury steroids consisting of high-dose methylprednisolone (NASCIS-2 [National Acute Spinal Cord Injury Study-2] protocol: $30 \mathrm{mg} / \mathrm{kg}$ bolus followed by $5.4 \mathrm{mg} / \mathrm{kg} / \mathrm{hr}$ for $23 \mathrm{hr})^{24,25}$. If the change in the evoked potentials was temporally associated with placement of a bone graft or internal fixation, surgical intervention included removal of the graft or fixation as well.

\section{Statistical Methods}

The accuracy of the monitoring with regard to detecting impending iatrogenic spinal cord injury was expressed by calculating sensitivity and specificity. We defined an impending injury as any important neurophysiological change that prompted some type of intervention (for example, increasing the mean arterial pressure, administering steroids, or removing bone graft). A true-positive result was defined as any case in which the changes in the evoked potentials were reversed immediately by the intervention or in which the changes persisted and the patient awoke with a new neurological deficit. A false-positive result was defined as any case in which the changes in the evoked potentials did not respond to intervention and the patient awoke neurologically intact. A true-negative result was defined as a case in which monitoring revealed no changes 
The Journal of Bone \& JOINT Surgery • JBIS. ORG VOLUME 86-A · NUMBER 6 · JUNE 2004
Transcranial EleCtRIC MOTOR AND SOMATOSENSORY Evoked Potentials in Cervical Spine Surgery and there was no new postoperative deficit. A false-negative result was defined as the new onset of a neurological deficit in a patient who had had no change in the neurophysiological monitoring data or in whom the change had resolved to a value within 2.0 standard deviations of the original baseline value following an intervention or by the end of the surgical procedure.

In addition, the Fisher exact probability test was used to assess whether the presence of cervical spondylotic myelopathy with or without ossification of the posterior longitudinal ligament was associated with changes demonstrated by spinal cord monitoring.

\section{Results}

Twelve (2.8\%) of the 427 procedures met the criteria for a 1 persistent neurophysiological change. The changes occurred in eight men and four women who ranged in age from thirty-nine to sixty-five years (mean, fifty-four years) at the time of the index procedure. Two of the twelve patients, one man and one woman, emerged from the anesthesia with a new neurological deficit. Hence, the point prevalence of iatrogenic neurological injury in this series of patients treated with cervical spine surgery was $0.47 \%$.

\section{Operating Characteristics}

The $2 \times 2$ contingency tables used to calculate the sensitivity and specificity of the tceMEP and SSEP monitoring are presented in Tables I and II. Both the sensitivity and the specificity of tceMEP monitoring were $100 \%$. The sensitivity of the SSEP monitoring, however, was only $25 \%$, although the specificity was $100 \%$. Of the twelve patients with substantial tceMEP amplitude change, two emerged from anesthesia with a motor deficit. One presented with a dense lower-extremity paraplegia, which did not improve subsequently, and the other had transient but clinically evident upper-extremity weakness. Both patients had complete acute bilateral or unilateral loss of the tceMEPs that never recovered, despite intraoperative intervention. Only one of these patients, however, had a concomitant SSEP amplitude loss, and that change lagged behind the onset of the tceMEP changes by thirty-three minutes.

Four additional patients had acute tceMEP amplitude loss of $80 \%$ or greater that necessitated surgical intervention. The precipitating etiology was attributed to spinal distraction immediately following insertion of a strut graft. In all four procedures, the tceMEP amplitudes reverted to near-baseline values only after the graft was removed. During three of the four procedures, there was a delay of three to seventeen minutes between the detection of changes by the tceMEP monitoring and the detection of changes by the SSEP monitoring. One patient, who had a complete loss of tceMEPs following insertion of a strut graft, had stable SSEP amplitudes, which were classified as a false-negative SSEP finding. In the remaining six patients, tceMEP amplitude changes responded to an increase of the mean arterial pressure to $90 \mathrm{~mm} \mathrm{Hg}$ or more and the administration of a methylprednisolone bolus. All six patients had completely stable, unchanged SSEP amplitudes.
TABLE I $2 \times 2$ Contingency Table Used to Calculate Sensitivity, Specificity, and Positive and Negative Likelihood Ratios for Transcranial Electric Motor Evoked Potential Monitoring*

\begin{tabular}{|lrcr|} 
& \multicolumn{3}{c|}{ Emerging Injury } \\
\cline { 2 - 4 } tceMEP & Yes & No & Total \\
\hline Yes & 12 & 0 & 12 \\
No & 0 & 415 & 415 \\
Total & 12 & 415 & 427 \\
\hline *See text for definition of operational characteristics. \\
\hline
\end{tabular}

TABLE II $2 \times 2$ Contingency Table Used to Calculate Sensitivity, Specificity, and Positive and Negative Likelihood Ratios for Somatosensory Evoked Potential Monitoring*

\begin{tabular}{|lccc|}
\hline & \multicolumn{3}{c|}{ Emerging Injury } \\
\cline { 2 - 4 } Change & Yes & No & Total \\
\hline Yes & 3 & 0 & 3 \\
No & 9 & 415 & 424 \\
Total & 12 & 415 & 427 \\
\hline * See text for definition of operational characteristics. \\
\hline
\end{tabular}

In the four patients in whom major changes were detected by both modalities, the SSEP changes lagged behind the tceMEP changes by three to thirty-three minutes. Hence, although the specificity of the SSEP monitoring was equivalent to that of the tceMEP monitoring, the temporal differences were clinically important.

\section{Exemplary Case}

A patient with cervical spondylotic myelopathy and ossification of the posterior longitudinal ligament underwent C4-C6 corpectomy with anterior strut-grafting from C3 to C7 (Figs. 1-A and 1-B). He had stable ulnar and posterior tibial nerve SSEPs throughout the multilevel decompression and placement of the strut graft, with no changes relative to the baseline (Fig. 1-A). On the basis of the SSEP recordings alone, this would have been considered an uneventful surgical procedure, with no untoward neurological consequences predicted. The tceMEP data (Fig. 1-B), however, demonstrated that, on placement of the strut graft, there was an acute loss of upper and lower-extremity motor potentials on the right side, accompanied by decreases in amplitude on the left side. This time-locked neurophysiological event prompted an immediate response to try to prevent the evolving spinal cord injury. Intervention included raising the mean arterial pressure to 93 $\mathrm{mm} \mathrm{Hg}$, removing the strut graft, and administering spinalcord-injury steroids. Shortly thereafter, the tceMEPs reappeared, and there was a continuous increase in amplitude toward baseline values over the course of the next ten minutes. 


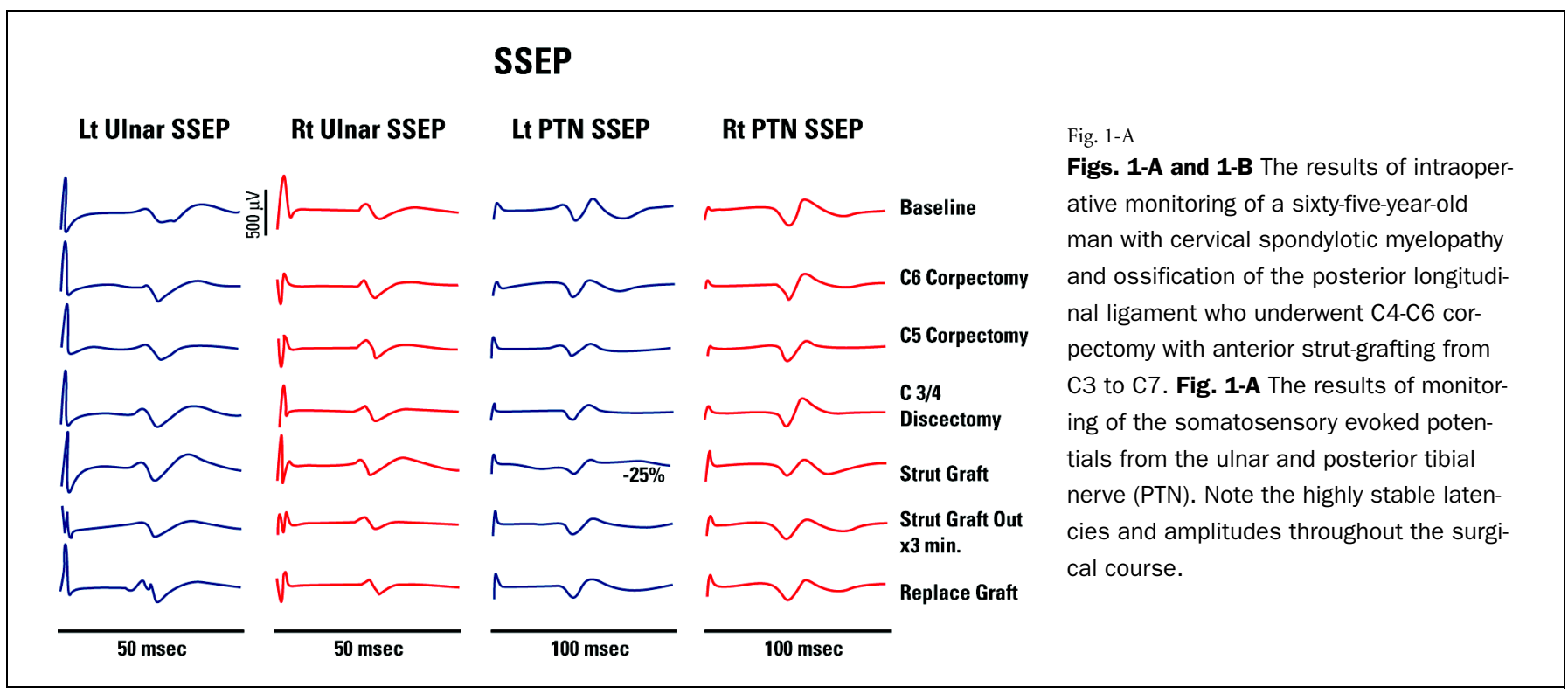

A shortened graft was reinserted, with no more events. The patient awoke without any new neurological deficits.

\section{Diagnostic Risk Factors}

Because of its frequency of occurrence, cervical spondylotic myelopathy with or without ossification of the posterior longitudinal ligament emerged as a prominent risk factor for iatrogenic injury during cervical spine surgery. The $2 \times 2$ contingency tables used to perform the Fisher exact probability test comparing patients with and without cervical spondylotic myelopathy and comparing those with and without ossification of the posterior longitudinal ligament are presented in Tables III and IV. The results of that analysis indicated a significantly greater likelihood $(p=0.0057)$ that patients with myelopathy would have intraoperative monitoring events than would patients without myelopathy. Similarly, the presence of ossification of the posterior longitudinal liga- ment was an additional risk factor, although the significance was weaker $(\mathrm{p}=0.02)$. Thus, among patients with myelopathy, those with ossification of the posterior longitudinal ligament were at a higher risk for emerging intraoperative spinal cord injury than were those without ossification of the posterior longitudinal ligament.

\section{Discussion}

any spine surgeons who use anterior cervical ap1 proaches have been reluctant to use SSEP monitoring, fearing a false-negative result because the SSEP response is mediated primarily from the dorsal sensory rather than the ventral motor tracts of the spinal cord. Selective injury to the anterior portion of the spinal cord may be a greater risk during anterior cervical spine surgery than it is during posterior scoliosis surgery. Monitoring the corticospinal tracts following transcranial electrical stimulation has shown great prom-

\section{TceMEPs}

Fig. 1-B

The results of monitoring of the transcranial electric motor evoked potentials from the first dorsal interosseous (FDI) and anterior tibialis (ATib) muscles. Note the acute loss of upper and lower-extremity motor potentials on the right on placement of the strut graft.
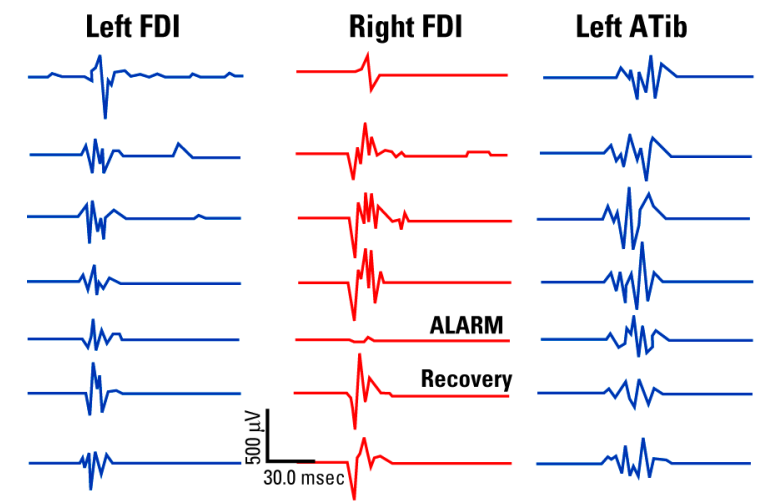
The Journal of Bone \& JOINT SURGERY • JBIS.ORG VOLUME 86-A · Number 6 · JUNE 2004
Transcranial EleCtRIC MOTOR AND SOMATOSENSORY Evoked Potentials in Cervical Spine Surgery

\begin{tabular}{|c|c|c|c|}
\hline \multirow{2}{*}{$\begin{array}{l}\text { Cervical } \\
\text { Spondylotic } \\
\text { Myelopathy }\end{array}$} & \multicolumn{3}{|c|}{ Neurophysiological Change } \\
\hline & Yes & No & Total \\
\hline Yes & 11 & 205 & 216 \\
\hline No & 1 & 210 & 211 \\
\hline Total & 12 & 415 & 427 \\
\hline
\end{tabular}

\begin{tabular}{|c|c|c|c|}
\hline \multirow{2}{*}{$\begin{array}{c}\text { Ossification of } \\
\text { the Posterior } \\
\text { Longitudinal Ligament }\end{array}$} & \multicolumn{3}{|c|}{ Neurophysiological Change } \\
\hline & Yes & No & Total \\
\hline Yes & 4 & 18 & 22 \\
\hline No & 7 & 187 & 194 \\
\hline Total & 11 & 205 & 216 \\
\hline
\end{tabular}

ise for the real-time identification of iatrogenic intraoperative injury to the descending spinal-motor pathway. To our knowledge, however, there has been no large-scale investigation to determine the sensitivity and specificity of tceMEPs compared with SSEPs for monitoring the function of the spinal cord during cervical spine surgery.

The clinical goal of any diagnostic modality is both high sensitivity (a low false-negative rate) and high specificity (a low false-positive rate); however, it is often difficult to achieve one without sacrificing the other. For example, May et al. ${ }^{26}$ reported a sensitivity of $99 \%$ at the cost of only $27 \%$ specificity with the use of SSEP monitoring in 191 patients undergoing cervical spine surgery. This suggests that defining a major change too liberally so that it encompasses the wide range of normal variability will result in a high false-positive rate in a population of patients with a low likelihood of neurological compromise. A high sensitivity (a low threshold for a remarkable change) can be disruptive to the surgical procedure if it produces too many false-positive results. Alternatively, too strict a cutoff criterion might decrease the likelihood of false-positive results but increase the possibility of false-negative findings.

Because of the statistical variability surrounding SSEP recordings and the influence of anesthesia on both SSEPs and tceMEPs ${ }^{23,27-31}$, we used a cutoff criterion of $60 \%$ or greater loss of amplitude (more than 2.0 standard deviations) and we carefully controlled the anesthesia, eliminating all inhalational agents. We found that tceMEP monitoring was both $100 \%$ sensitive and $100 \%$ specific. It is important to note that, in all procedures during which a major neurophysiological change resulted in a surgical alert, the tceMEP amplitude change was $80 \%$ or greater. Moreover, of the twelve patients considered to have had a tceMEP change, ten responded favorably to intervention, with prompt neurophysiological improvement, and all ten awoke with no untoward deficit. Conversely, the two patients who had postoperative paralysis had not had such improvement after intraoperative intervention. The sensitivity of SSEP monitoring, with use of a cutoff criterion that accounted for inherent variability, was unacceptably low (25\%), although the specificity was $100 \%$. This high false-negative rate for injury detection justifies the lack of enthusiasm that most surgeons have for monitoring cervical spine surgery with SSEPs. Indeed, Jones et al. ${ }^{32}$ recently reported two cases of quadriparesis following anterior cervical discectomy despite normal, unchanged SSEPs.

Another important finding was the temporal relationship between tceMEP and SSEP amplitude changes. During the four procedures in which SSEP amplitude changed in concordance with the changes in the tceMEPs, the SSEP signal alterations lagged behind those of the tceMEPs by an average of sixteen minutes. In fact, one of the two patients who awoke with new-onset paralysis had a thirty-three-minute delay between the tceMEP alert and the SSEP detection. Such a delay between injury evolution and monitoring alert reduces the window of opportunity for intervention and may prevent or compromise reversal of spinal cord injury.

An additional factor that appeared to play a critical role was the mean arterial blood pressure and its effect on spinal cord perfusion pressure. In six of the twelve patients with a major tceMEP change, the monitoring amplitudes reverted to baseline within five minutes of raising the mean arterial pressure to 90 $\mathrm{mm} \mathrm{Hg}$ or more. Another four of the twelve cases of tceMEP changes occurred during strut-grafting, suggesting that an oversized graft may have caused excessive distraction with stretching of the vascular supply of the spinal cord. Clearly, maintenance of adequate spinal cord perfusion is important during cervical spine surgery, particularly at times when stress is placed on the spinal cord, such as during decompression and strut-grafting. When a loss of tceMEP amplitude is noted during a procedure, we recommend raising the mean arterial pressure to at least 90 $\mathrm{mm} \mathrm{Hg}$ prior to strut-graft revision and reinsertion.

In this study, we also identified preoperative patientrelated risk factors associated with changes demonstrated by intraoperative monitoring. All but one of the twelve patients who had tceMEP changes had a preoperative diagnosis of cervical spondylotic myelopathy, and four of them also presented with ossification of the posterior longitudinal ligament. Statistical analysis demonstrated that the likelihood of intraoperative monitoring detecting changes was greater in patients with myelopathy in general, and in those with ossification of the posterior longitudinal ligament in particular, than it was in patients who did not have myelopathy. It would appear, therefore, that special attention must be given to ensuring adequate spinal cord perfusion in these patients.

\section{Appendix}

A table showing the anesthesia protocol used during the eA study period is available with the electronic versions of this article, on our web site at www.jbjs.org (go to the article citation and click on "Supplementary Material") and on our 
The Journal of Bone \& JOINT SURGERY · JBJS.ORG Volume 86-A · Number $6 \cdot$ June 2004
Transcranial Electric Motor and Somatosensory

Evoked Potentials in Cervical Spine Surgery quarterly CD-ROM (call our subscription department, at 781449-9780, to order the CD-ROM).

NOTE: The authors thank Evelyn Redtree, MS, CCC-A, for her data collection and analysis and for her editorial expertise in preparing this manuscript for publication.

Alan S. Hilibrand, MD

Venkat Sethuraman, MD

Alexander R. Vaccaro, MD

Todd J. Albert, MD

The Rothman Institute, 925 Chestnut Street, 5th Floor, Philadelphia, PA 19107
Daniel M. Schwartz, PhD, DABNM

Surgical Monitoring Associates, 25 Bala Avenue, Suite 105, Bala Cynwyd, PA 19004. E-mail address: danielmschwartz@mac.com

The authors did not receive grants or outside funding in support of their research or preparation of this manuscript. They did not receive payments or other benefits or a commitment or agreement to provide such benefits from a commercial entity. No commercia entity paid or directed, or agreed to pay or direct, any benefits to any research fund, foundation, educational institution, or other charitable or nonprofit organization with which the authors are affiliated or associated.

\section{References}

1. Nuwer MR, Dawson EG, Carlson LG, Kanim LE, Sherman JE. Somatosensory evoked potential spinal cord monitoring reduces neurologic deficits after scoliosis surgery: results of a large multicenter survey. Electroencephalogr Clin Neurophysiol. 1995;96:6-11.

2. Ecker ML, Dormans JP, Schwartz DM, Drummond DS, Bulman WA. Efficacy of spinal cord monitoring in scoliosis surgery in patients with cerebral palsy. J Spinal Disord. 1996;9:159-64.

3. Schwartz DM, Drummond DS, Schwartz JA, Wierzbowski LR, Sestokas AK, Pratt RE Jr, Turner LA. Neurophysiological monitoring during scoliosis surgery: a multimodality approach. Semin Spine Surg. 1997;9:97-111.

4. Noordeen MH, Lee J, Gibbons CE, Taylor BA, Bentley G. Spinal cord monitoring in operations for neuromuscular scoliosis. J Bone Joint Surg Br. 1997; 79:53-7.

5. Padberg AM, Bridwell KH. Spinal cord monitoring: current state of the art. Orthop Clin North Am. 1999;30:407-33, viii.

6. Luk KD, Hu Y, Wong YW, Cheung KM. Evaluation of various evoked potentia techniques for spinal cord monitoring during scoliosis surgery. Spine. 2001; 26:1772-7.

7. Drummond DS, Schwartz DM, Johnston DR, Farmer JF. Neurological injury complicating surgery. In: DeWald RL, editor. Spinal deformities: the comprehensive text. New York: Thieme; 2003. p 615-25.

8. Ginsberg HH, Shetter AG, Raudzens PA. Postoperative paraplegia with preserved intraoperative somatosensory evoked potentials. Case report. J Neurosurg. 1985;63:296-300.

9. Ben-David B, Haller G, Taylor P. Anterior spinal fusion complicated by paraplegia. A case report of a false-negative somatosensory-evoked potential. Spine. 1987;12:536-9.

10. Dinner DS, Luders H, Lesser RP, Morris HH, Barnett G, Klem G. Intraoperative spinal somatosensory evoked potential monitoring. J Neurosurg. 1986;65:807-14.

11. Lesser RP, Raudzens PA, Luders H, Nuwer MR, Goldie WD, Morris HH 3rd, Dinner DS, Klem G, Hahn JF, Shetter AG, Ginsburg HH; Gurd AR. Postoperative neurological deficits may occur despite unchanged intraoperative somatosensory evoked potentials. Ann Neurol. 1986;19:22-5.

12. Zornow MH, Grafe MR, Tybor C, Swenson MR. Preservation of evoked potentials in a case of anterior spinal artery syndrome. Electroencephalogr Clin Neurophysiol. 1990;77:137-9.

13. Bridwell KH, Lenke LG, Baldus C, Blanke K. Major intraoperative neurologic deficits in pediatric and adult spinal deformity patients. Incidence and etiology at one institution. Spine. 1998;23:324-31.

14. Burke D, Hicks R, Stephen J, Woodforth I, Crawford M. Assessment of corticospinal and somatosensory conduction simultaneously during scoliosis surgery. Electroencephalogr Clin Neurophysiol. 1992;85:388-96.

15. Jones SJ, Harrison R, Koh KF, Mendoza N, Crockard HA. Motor evoked potential monitoring during spinal surgery: responses of distal limb muscles to transcranial cortical stimulation with pulse trains. Electroencephalogr Clin Neurophysiol. 1996;100:375-83.

16. Schwartz DM, Sestokas AK, Turner LA, Morledge DE, DiNardo AA Jr, Beacham SG. Neurophysiological identification of iatrogenic neural injury during complex spine surgery. Semin Spine Surg. 1998;10:242-51.

17. Schwartz DM, Wierzbowski LR, Fan D, Sestokas AK. Surgical neurophysiologic monitoring. In: Vaccaro AR, Betz RR, Zeidman SM, editors. Principles and practice of spine surgery. Philadelphia, PA: Mosby; 2003. p 115-26.

18. Langeloo DD, Journee HL, Polak B, de Kleuver M. A new application of TCE-
MEP: spinal cord monitoring in patients with severe neuromuscular weakness undergoing corrective spine surgery. J Spinal Disord. 2001;14:445-8.

19. MacDonald DB, AI Zayed Z, Khoudeir I, Stigsby B. Monitoring scoliosis surgery with combined multiple pulse transcranial electric motor and cortical somatosensory-evoked potentials from the lower and upper extremities. Spine. 2003;28:194-203.

20. DiCindio S, Theroux M, Shah S, Miller F, Dabney K, Brislin RP, Schwartz D. Multimodality monitoring of transcranial electric motor and somatosensoryevoked potentials during surgical correction of spinal deformity in patients with cerebral palsy and other neuromuscular disorders. Spine. 2003;28: 1851-6.

21. Fan D, Schwartz DM, Vaccaro AR, Hilibrand AS, Albert TJ. Intraoperative neurophysiologic detection of iatrogenic $C 5$ nerve root injury during laminectomy for cervical compression myelopathy. Spine. 2002;27:2499-502.

22. Schwartz DM. Intraoperative neurophysiological monitoring during posttraumatic spine surgery. In: Vaccaro AR, editor. Fractures of the cervical, thoracic, and lumbar spine. New York: Marcel Dekker; 2003. p 373-83.

23. York DH, Chabot RJ, Gaines RW. Response variability of somatosensory evoked potentials during scoliosis surgery. Spine. 1987;12:864-76.

24. Bracken MB, Shepard MJ, Collins WF Jr, Holford TR, Young W, Baskin DS, Eisenberg HM, Flamm E, Leo-Summers L, Maroon J, Marshall LS, Perot PL Jr, Piepmeier J, Sonntag VKH, Wagner FC, Wilberger JE, Winn HR. A randomized, controlled trial of methylprednisolone or naloxone in the treatment of acute spinal-cord injury. Results of the Second National Acute Spinal Cord Injury Study. N Engl J Med. 1990;322:1405-11.

25. Bracken MB, Shepard MJ, Holford TR, Leo-Summers L, Aldrich EF, Fazl M, Fehlings M, Herr DL, Hitchon PW, Marshall LF, Nockels RP, Pascale V, Perot PL Jr, Piepmeier J, Sonntag VK, Wagner F, Wilberger JE, Winn HR, Young W. Administration of methylprednisolone for 24 or 48 hours or tirilazad mesylate for 48 hours in the treatment of acute spinal cord injury. Results of the Third National Acute Spinal Cord Injury Randomized Controlled Trial. National Acute Spinal Cord Injury Study. JAMA. 1997;277:1597-604.

26. May DM, Jones SJ, Crockard HA. Somatosensory evoked potential monitoring in cervical surgery: identification of pre- and intraoperative risk factors as sociated with neurological deterioration. J Neurosurg. 1996;85:566-73.

27. Gugino LD, Schwartz DM. Monitoring motor evoked potentials. Read at the Annual Meeting of the Society of Neurosurgical Anesthesia and Critical Care; 2001 Oct 12; New Orleans, LA.

28. Kombos T, Suess 0, Da Silva C, Ciklatekerlio O, Nobis V, Brock M. Impact of somatosensory evoked potential monitoring on cervical surgery. J Clin Neurophysiol. 2003;20:122-8.

29. Lubicky JP, Spadaro JA, Yuan HA, Fredrickson BE, Henderson N. Variability of somatosensory cortical evoked potential monitoring during spinal surgery. Spine. 1989;14:790-8.

30. Noonan KJ, Walker T, Feinberg JR, Nagel M, Didelot W, Lindseth R. Factors related to false- versus true-positive neuromonitoring changes in adolescent idiopathic scoliosis surgery. Spine. 2002;27:825-30.

31. Schwartz DM, Schwartz JA, Pratt RE Jr, Wierzbowski LR, Sestokas AK. Influence of nitrous oxide on posterior tibial nerve cortical somatosensory evoked potentials. J Spinal Disord. 1997;10:80-6.

32. Jones SJ, Buonamassa S, Crockard HA. Two cases of quadriparesis following anterior cervical discectomy, with normal perioperative somatosensory evoked potentials. J Neurol Neurosurg Psychiatry. 2003;74:273-6.

33. Schwartz DM. Intraoperative neurophysiological monitoring during cervical spine surgery. Oper Tech Orthop. 1996;6:6-12 